\section{ESTIMATIVA DO VOLUME RUMINAL DE BEZERROS COM EMPREGO DO POLIETILENOGLICOL*}

\author{
CARLOS DE SOUSA LUCCI \\ Professor Adjunto
}

Faculdade de Medicina Veterinária e Zootecnia da USP

HARRY RUSSEL CONRAD

Professor da Universidade Estadual de Ohio, EUA

BURK DEHORITY

Pesquisador PHA

Universidade Estadual de Ohio, EUA

SUSAN C. PEYTON

Pesquisador PHA

Universidade Estadual de Ohio, EUA

SUE K. BIELAWSKI

Pesquisador M.S.

Universidade Estadual de Ohio, EUA

JEAN A. GRUBB

Pesquisador M.S.

Universidade Estadual de Ohio, EUA

LUCCI, C.S.; CONRAD, H.R.; DEHORITY, B.; PEYTON, S.C. BIELAWSKI, S.K.; GRUBB, J.A. Estimativa do volume ruminal de bezerros com emprego do polietilenoqlicol. Fac. Med. vet. Zootec. Univ. S. Paulo, 19(2): 163-6, 1982.

RESUMO: Oito bezerros Holandeses tiveram seus volumes ruminais estimados com emprego de polietilinoglicol (PEG) e em segulda. sacrificados para avaliação dessas estimativas. Fol desenvolvida uma técnica de amostragem de material do rúmen via sonda esofagiana. Os dados obtidos mostraram não ser possivel estimar com precisão o volume ruminal através desta técnica, devido a baixa correlação apresentada entre volumes ruminais e pesos dos con. teúdos após o sacrifício $(r=0,292)$. As correlações entre: consumo de alimentos $x$ pesos dos conteúdos ruminais; consumo de alimentos $x$ pesos de bezerros; e pesos dos bezerros e conteúdos ruminais, foram significativos.

UNITERMOS: Bezerros "; Nutrição, ruminantes *; Rúmen, volume *

\section{INTRODUÇÃO}

O conhecimento do volume líquido do rúmen de bezerros, sem necessidade de fistulação ou abate, seria de extrema utilidade em estudos de nutrição. Sendo anima is em fase de desenvolvimento acelerado dos proventrículos, descarta-se a possibilidade de execução de fístulas, que permitiriam colheitas de amostras representativas dos conteúdos ruminais, importantes ao se trabalhar com marcadores, como o polietilenoglicol (PEG).

Desde 1953 SPERBER e cols ${ }^{3}$ introduziram o PEG como substância marcadora em estudos com ruminantes. STIG HYDEN ${ }^{3}$, posteriormente, melhorou a técnica de determinação do PEG em laboratório, tornando o processo todo mais rápido e eficaz.

ULYATT e cols 6 demonstraram haver precisão elevada da estimativa de volume ruminal, em ovinos fistulados, com o emprego do PEG. Também HYDEN 4 chegou às mesmas conclusões, trabalhando com ovinos e caprinos. BAUMAN e cols ${ }^{1}$ obtiveram sucesso trabalhando com vacas fistuladas, sob diferentes dietas (com predomínio de concentrados ou predomínio de volumosos). Segundo estes últimos autores, rações assim diversificadas não influiram significativamente nos volumes dos rumens.

Todos os autores que estimaram volumes de rúmen com emprego do PEG, o fizeram em animais fistulados. O presente trabalho foi uma tentativa de criar uma técnica de estimar volume ruminal em bezerros jovens, não fistulados, quando a fistulação do rúmen seria impossível de se realizar sem maiores inconvenientes.

\section{MATERIAL E MÉTODO}

Oito bezerros Holandeses, var. malhada de preto, machos, com 8 semanas de idade, tiveram os volumes de seus rúmens avaliados com o uso do PEG, conforme procedimento descrito por DEHORITY2, para carneiros fistulados. No caso presente, não sendo os animais providos de fístulas ruminais, foi empregada uma sonda esofagiana. Utilizou-se 10 gramas de PEG por bezerros, com $40 \mathrm{ml}$ de água distilada. Amostras de conteúdo do rúmen foram colhidas: imediatamente antes de adicionar PEG; uma hora após e vinte e quatro horas após. O PEG foi posto no interior do rúmen com auxílio de seringa hipodérmica de $40 \mathrm{ml}$ de capacidade, conectada a um tubo de plástico que corria por dentro da sonda esofagiana. Após colocar-se o marcador, mais $40 \mathrm{ml}$ de água distilada eram adicionados à seringa $\mathrm{e}$ injetados no interior do proventrículo, para perfeita lavagem dos resíduos da solução com marcador.

Imediatamente após a colheita de material do rúmen, executada 24 horas depois da injeção de PEG, os bezerros eram sacrificados por eletrocução. Em seguida, atravês de laparotomia os ventrículos gástricos foram removidos, com o cuidado de antes fazer-se ligaduras na porção terminal do esôfago e entre rúmen e omaso, para manter-se intactos os conteúdos totais dos rúmens-retículos. 
Abertos os rúmens, seus conteúdos eram completamente evacuados e reunidos em recipientes limpos e secos. para pesagem.

O regime alimentar dos bezerros tor padronizado para: colostro até 3 dias de idade, depois leite $(4 \mathrm{~kg} / \mathrm{dia})$ e mistura iniciadora até os 28 dias, quando se praticava $\checkmark$ desaleitamento. A partir dai, os bezerros recebiam apenas Mistura iniciadora (máximo de $2,5 \mathrm{~kg} /$ animal/dia) e feno de alfafa inteiro, sem picar, "ad libitum".

Este experimento foi conduzido no Centro de Pesquisa e Desenvolvimento em Agricultura de Wooster, pertencen- te à Universıdade Estadual de Ohio, Estados Unidos da América, no segundo semestre de 1978.

\section{RESULTADOS E DISCUSSÃO}

A tabela 1 apresenta os resultados obtidos como: volumes ruminais (estimados em vida), pesos dos conteúdos ruminais (obtidos após o abate), pesos vivos dos bezerros, teores de matéria seca do conteúdo ruminal, e ainda as ingestões de alimentos nas últimas 24 horas de vida (pesos da mistura iniciadora mais feno).

TABELA 1 - Pesos dos bezerros, e consumos de alımentos, em quilogramas; volumes dos rúmens, em litros; pesos dos conteúdos, em quilogramas e matéria seca dos conteúdos, em porcentagens, Ohio, EUA, 1978.

\begin{tabular}{|c|c|c|c|c|c|}
\hline $\begin{array}{l}\text { Bezerro } \\
\text { no }\end{array}$ & $\begin{array}{l}\text { Pesos } \\
\text { dos } \\
\text { bezerros } \\
\text { kg }\end{array}$ & $\begin{array}{c}\text { Consumo } \\
\text { Alimentos } \\
\text { kg }\end{array}$ & $\begin{array}{c}\text { Volumes } \\
\text { dos } \\
\text { rúmens } \\
\text { I }\end{array}$ & $\begin{array}{l}\text { Pesos } \\
\text { dos } \\
\text { conteúdos } \\
\quad \mathrm{kg}\end{array}$ & $\begin{array}{l}\text { Matéria } \\
\text { seca } \\
\text { conteúdos } \\
\quad \%\end{array}$ \\
\hline 1 & 83 & 5,8 & 13,1 & 9,7 & 15,8 \\
\hline 2 & 49 & 4,1 & 3,2 & 4,7 & 15,8 \\
\hline 3 & 32 & 4,2 & 13,0 & 3,5 & 15,3 \\
\hline 4 & 60 & 3,5 & 3,0 & 5,5 & 14,9 \\
\hline 5 & 80 & 7,3 & 14,8 & 7,9 & 15,8 \\
\hline 6 & 75 & 6,2 & 2,2 & 8,2 & 16,3 \\
\hline 7 & 68 & 6,3 & 12,6 & 9,0 & 17,3 \\
\hline 8 & 79 & 6,3 & 9,1 & 7,6 & 15,3 \\
\hline
\end{tabular}


A tabela 2 apresenta correlações entre algumas variáveis observadas neste trabalho.

TABELA 2 - Correlações ( $r$ ) entre as variáveis: volume do rúmen, peso do conteúdo, consumo de alimentos e peso do bezerro

\begin{tabular}{|c|c|c|c|c|}
\hline VARIẢVEIS & $\begin{array}{l}\text { VOLUMES } \\
\text { DOS } \\
\text { RÜMENS }\end{array}$ & $\begin{array}{l}\text { PESOS } \\
\text { DOS } \\
\text { CONTEÚDOS }\end{array}$ & $\begin{array}{l}\text { CONSUMO } \\
\text { DOS } \\
\text { ALIMENTOS }\end{array}$ & $\begin{array}{l}\text { PESOS } \\
\text { DOS } \\
\text { BEZERROS }\end{array}$ \\
\hline $\begin{array}{l}\text { VOLUMES } \\
\text { DOS } \\
\text { RÚMENS }\end{array}$ & - & 0,292 & 0,496 & 0,146 \\
\hline $\begin{array}{l}\text { PESOS } \\
\text { DOS } \\
\text { CONTEŨDOS }\end{array}$ & 0,292 & - & $0,774\left({ }^{*}\right)$ & $\left.0,9081^{* *}\right)$ \\
\hline $\begin{array}{l}\text { CONSUMOS } \\
\text { DOS } \\
\text { ALIMENTOS }\end{array}$ & 0,496 & $0,774\left(^{*}\right)$ & - & $\left.0,764 \quad i^{*}\right)$ \\
\hline $\begin{array}{l}\text { PESOS } \\
\text { DOS } \\
\text { BEZERROS }\end{array}$ & 0,146 & $0,908\left({ }^{* *}\right)$ & $0,764\left({ }^{*}\right)$ & - \\
\hline
\end{tabular}

$$
\begin{aligned}
* & =p \leqslant 0,05 \\
* * & =p \leqslant 0,01
\end{aligned}
$$

A correlação mais elevada $(r=0,908)$ encontrada entre pesos dos bezerros e pesos dos conteúdos ruminais, foi considerada como altamente significativa ( $p 0,01$ ). Correlações significativas (p 0,05 ) também foram encontradas entre pesos dos bezerros e consumos de alimentos ( $r=0,764)$ e entre consumos de alimentos e pesos dos conteúdos $(r=0,774)$, todas elas facilmente explicáveis. A relação entre volumes estimados dos rúmens e pesos dos conteúdos, foi decepcionantemente baixa $(r=0,292)$.

A tentativa de avaliar-se volumes ruminais com emprego de PEG, em bezerros não fistulados, segundo estes resultados, não obteve sucesso. Provavelmente, a falha da técnica resida no fato dos conteúdos ruminais dos bezerros não sofrerem boa homogeinização apenas com os movimentos das paredes deste proventriculo. Anote-se que todos os dados foram obtidos com animais que apresentavam com toda plenitude o ato da ruminação, e ainda que o feno foi ministrado inteiro, sem picar, para provocar uma mistura melhor dos conteúdos ruminais.
LUCCI, C.S.; CONRAD, H.R.; DEHORITY, B.; PEYTON, S.C.; BIELAWSKI, S.K.; GRUBB, J.A. Calves's rumen volumes, estimated through polvetilenglicol (PEG). Rev. Fac. Med, vet. Zootec.Univ.S.Paulo, 19(2): 1636, 1982.

SUMMARY: Eight Holstein calves had their rumens's volumes estimated through polyetilenglicol (PEG) utilization; animals were next, slaughtered to evaluate the estimations. A technique was developed to colect rumen contents by aesophagel canula. Data showed this technique must be improved, because the correlation between estimated rumen volumes and weight of rumen contents was low $(r=0,292)$. Correlations between: feed consumption $x$ weight of rumen's contents; feed consumption $x$ calve's weight; calve's weight $x$ rumen contents, were significant.

UNITF RMS: Calves*, Nutrition of ruminants"; Rumun volume* 


\section{REFERENCIAS BIBLIOGRAFICAS}

1 - BAUMAN, D.E.; DAVIS, C.L.; FROBISH, R.A.; SCHAN, D.S. Evaluation of polyethyleneglicol method in determining rumen fluid volume in dairy cows fed different diets. J. Dairy Sci., 54: $928,1971$.

2 - DEHORITY, B. [Curso de pós-graduação em microbiologia do rúmen.] Wooster, State University of Ohio, s.d. 4 p. (Apostila).

3- HYDEN, S. A turbidimetric method for the determination of higher polyethyleneglicol in biological materials. Ann. Roy. Agric. Coll. Sweden, 22:139, 1955.
4-HYDEN, S. Determination of the amount of fluid in the reticulorumen of the sheep and its rate of passage to the omasum, kg. Lantbr. Högsk. Ann. 27:51, 1961.

5 - SPERBER, I.; HYDEN, S.; EKAMAN, J. The use of polyethyleneglicol as a reference substance in the of ruminant digestion. Ann. Roy. Agric. Coll. Sweden, 20:337, 1953.

6 - ULYATT, J.; BLAXTER, K.L.; McDONALD, I. The relations between the apparent digestibility of roughages in the rumen and lower gut of sheep. the volume of fluid in the rumen and voluntary feed intake. Anim. Prod., 9:463, 1927.

Recebido para publicação em: 07-06-82. Aprovado para publicação em: 18-11-82. 